\title{
Prolongation de la clause du besoin: informations pour les médecins qui n'ont pas encore ouvert de cabinet*
}

Hanspeter Kuhn, secrétaire général adjoint de la FMH

* Remarque: ce texte ne se veut pas un commentaire politique, mais uniquement une information destinée aux médecins concernés.

1 Ce texte de loi est en vigueur depuis le $1^{\text {er }}$ janvier 2005: www. admin.ch/ch/f/rs/832_10/ a55a.html.

2 Communiqué de presse: www.admin.ch/cp/f/429432ff $1 @$ fwsrvg.html; pour consulter l'ordonnance: www.bag.admin.ch/ kv/projekte/f/index.htm.

Correspondance:

Me Hanspeter Kuhn

$\mathrm{FMH}$

Elfenstrasse 18

Case postale 170

CH-3000 Berne 15

Tél. 0313591111

Fax 0313591112

E-mail secrétariat du service juridique: lex@hin.ch.
La clause du besoin est en vigueur depuis 2002. Par une révision intermédiaire de la LAMal datée du 8 octobre 2004, le Parlement a donné au Conseil fédéral la possibilité de prolonger cette clause pour une durée de trois ans. Le Parlement a en outre prévu que les admissions à pratiquer non utilisées devraient expirer dans un délai à fixer par le Conseil fédéral. L'art. 55a LAMal révisé [1] a la teneur suivante (compléments d'octobre 2004 mis en italique):

Art. 55a LAMal Limitation de l'admission de pratiquer à la charge de l'assurance-maladie

1 Le Conseil fédéral peut, pour une durée limitée à trois ans au plus, faire dépendre de la preuve d'un besoin l'admission des fournisseurs de prestations à pratiquer à la charge de l'assurance obligatoire des soins au sens des art. 36 à 38. Il fixe les critères correspondants. Il peut renouveler cette mesure une fois.

2 Les cantons et les fédérations de fournisseurs de prestations et d'assureurs doivent être consultés au préalable.

3 Les cantons désignent les fournisseurs de prestations conformément à l'al. 1 .

4 L'admission, une fois délivrée, expire lorsqu'il n'en est pas fait usage pendant un certain délai. Le Conseil fédéral fixe les conditions.

\section{La clause du besoin est en vigueur jusqu'au 3 juillet 2008 au plus tard}

Le 25 mai 2005, le Conseil fédéral a prolongé le blocage de l'accès à la pratique privée pour une durée de trois ans au maximum [2]. L'ordonnance révisée entrera en vigueur le 4 juillet 2005. Elle le restera jusqu'au 3 juillet 2008 au plus tard (ou jusqu'à la mise en application d'une solution de rechange dans le cadre d'une LAMal révisée).

\section{Expiration des admissions déjà octroyées: quand? 4 janvier ou 4 juillet 2006}

Le délai d'expiration fixé par le Conseil fédéral pour les admissions à pratiquer déjà obtenues est de 6 mois à partir de l'entrée en vigueur de la révision. Ce délai court donc jusqu'au 3 janvier 2006 (art. 3a al. 1 en relation avec l'art. 5 de l'ordonnance révisée).

Le Conseil fédéral a également prescrit que les cantons

- peuvent prolonger ce délai à 12 mois de façon générale, donc jusqu'au 3 juillet 2006 (art. 3, al. 2 de l'ordonnance révisée) et

- peuvent prolonger ce délai au-delà de la période normale prévue de 6 ou 12 mois, ce dans des cas particuliers et sur demande du médecin concerné. La condition posée est que le délai ne puisse pas être respecté «pour de justes motifs, en particulier pour des raisons de maladie, de maternité ou de formation postgraduée» (art. 3, al. 3 de l'ordonnance révisée).

Expiration des admissions déjà octroyées: qui est concerné? Selon la volonté du Conseil fédéral, les nombreux médecins qui avaient demandé leur autorisation d'exercer avant le 4 juillet 2002 et ne I'ont pas utilisée à ce jour sont aussi touchés - reste à savoir quelle sera la pratique des cantons

Cette question s'est avérée peu claire et controversée lors de la procédure de consultation. En péjorant encore le mauvais projet d'ordonnance de naguère, projet sur la base duquel des conseils juridiques avaient été donnés, le Conseil fédéral a maintenant précisé comment il entend faire interpréter la loi: selon son souhait, les quelque 2000 médecins qui, avant le 4 juillet 2002, avaient demandé une autorisation cantonale d'exercer et l'ont obtenue avant ou après cette date, risquent eux aussi de voir ladite autorisation devenir caduque s'ils n'en font pas usage. 


\section{Commentaire}

On ne sait pas encore si cette question sera un jour soumise à une révision judiciaire. Quelques interventions au Parlement abondent dans le sens du Conseil fédéral et de son interprétation du texte de la LAMal, mais le libellé de l'art. 55a LAMal parle en défaveur de cette interprétation: avant le 4 juillet 2002, il n'y avait en effet pas encore d'admissions à pratiquer, mais uniquement des autorisations d'exercer. Il est difficile de prédire ce que le juge déciderait ou décidera en la matière.

Il est toutefois clair qu'en définitive, ce sont les cantons qui tiennent les commandes en ce qui concerne la façon de lire et d'interpréter la loi: selon celle-ci, la Confédération fixe les «critères» (art. 55a, $1^{\text {er }}$ al. LAMal) alors que les cantons les appliquent (al. 3). Des cantons romands ont ainsi annoncé, au cours d'une consultation préliminaire, qu'ils entendent se conformer au libellé de la loi et ne pas faire expirer les autorisations d'exercer demandées avant le 4 juillet 2002, ne fût-ce que parce qu'ils n'ont pas intérêt à voir leurs médecins-assistants et chefs de cliniques expérimentés se mettre précipitamment à leur compte avant l'expiration du délai, ce afin de ne pas perdre leurs droits.

\section{Ce qui reste inchangé: les cantons sont en fin de compte libres de décider si et comment ils appliqueront la clause du besoin (ce qui atténue le problème des délais)}

3 «Art. 2 Aménagement du régime par les cantons

I Les cantons peuvent prévoir que: a. le nombre limite fixé à l'annexe 1 ne vaut pas pour une ou plusieurs catégories; b. dans une ou plusieurs catégories, aucune admission à pratiquer à la charge de l'assurancemaladie obligatoire ne sera délivrée tant que la densité médicale du canton concerné, telle qu'elle résulte de l'annexe 2, sera supérieure à celle de la région à laquelle ce canton est rattaché selon l'annexe 2 , ou supérieure à celle de la Suisse.

II Ils tiennent compte de la couverture sanitaire existant dans les cantons voisins et dans la région à laquelle ils sont rattachés selon l'annexe 2, et en Suisse.» www.admin.ch/ch/f/rs/832 103/a2.html

4 Commentaires relatifs au projet d'ordonnance, mars 2005, page 2 .

5 Explications du Conseil fédéral du 25 mai 2005, dernière page.

6 Bulletin officiel no 29, septembre 2004, page 1507 .

Le Conseil fédéral $n^{\prime} a$ pas modifié l'article 2 de l'ordonnance [3]. Ce qu'il avait annoncé dans les commentaires relatifs au projet d'ordonnance reste ainsi en vigueur:

«3. Principes de l'ordonnance

Les principes de l'ordonnance actuellement en vigueur sont maintenus. Ainsi, en vertu de l'art. 2, al. 1, let. a, de l'ordonnance, les cantons peuvent prévoir que le nombre limite fixé à l'annexe 1 ne s'applique pas à une ou plusieurs catégories de fournisseurs de prestations. Lorsqu'une catégorie de fournisseurs de prestations est soumise à limitation, les cantons peuvent, selon l'art. 3, admettre un nombre supérieur de fournisseurs si la couverture sanitaire est insuffisante dans cette catégorie. Ce critère permet aux cantons de délivrer en tout temps de nouvelles autorisations, malgré le gel des admissions, p.ex. lorsque la couverture sanitaire est insuffisante dans une région, notamment dans une région agricole.» [4]
Dans l'annexe, le Conseil fédéral a revu les chiffres maximaux. Ceux-ci ne constituent toutefois pas une limite fixe pour les cantons «mais servent d'indicateurs aux cantons qui procèdent aux évaluations correspondantes». [5]

En d'autres termes, les cantons sont libres, aujourd'hui mais aussi après le 4 juillet 2005, de décider si et comment ils appliqueront la clause du besoin: ils peuvent renoncer à cet outil pour l'ensemble de la médecine ou pour certaines disciplines en particulier.

\section{Reprise d'un cabinet individuel par deux médecins à temps partiel? Les cantons disposent}

Le Parlement avait exigé que le Conseil fédéral rende possible la reprise conjointe d'un cabinet individuel par plus d'un médecin, en arguant du fait que, comme on le sait, les modèles de temps de travail et d'organisation de la famille sont en pleine évolution. Le Conseiller fédéral Couchepin avait également donné son accord, ce que montre l'intervention de Mme Liliane Maury Pasquier (PS, GE):

«Nous souhaitons toutefois que cette mesure soit mise en œuvre sans pénaliser les médecins qui sont prêts, par exemple, à reprendre à plusieurs le cabinet d'un médecin partant à la retraite - donc avec une certaine souplesse. Lors des débats en commission, le Conseiller fédéral Couchepin nous a assuré que tel serait bien le cas et je pense qu'il nous le confirmera tout à l'heure.» [6]
Or le Conseil fédéral ne s'est pas soucié de tenir parole ni même de chercher une solution. Il ignore tout simplement ce point dans le texte de l'Ordonnance et dans ses explications du 25 mai. (Cette attitude n'encourage pas les jeunes médecins à se fier aux promesses du Conseil fédéral. Chaque pays aura à long terme la médecine qu'il aura méritée; les patients ne manqueront pas d'en remercier les politiques le jour venu ...)

Par conséquent, ce que le Conseil fédéral a écrit dans ses commentaires lors de la présentation du projet en mars 2005 demeure déterminant, à savoir qu'il transmet aux cantons le mandat d'«observer une certaine souplesse»:

«Comme cela a déjà été relevé en introduction, la présente ordonnance établit des règles pour une durée limitée. Or, ne seraitce que pour décider comment on pourrait définir une occupation à plein temps - 
7 Commentaires relatifs au projet d'ordonnance, page 6 , ch. 3.1.3.

8 Les adresses des directions de la santé se trouvent à l'adresse suivante: www.fmh.ch/ww/fr/ pub/awf/weiterbildung/europe/ annexe $4 . h$ tm p.ex. d'après un nombre d'heures à effectuer ou selon le montant des factures à la charge de la LAMal -, il est nécessaire de répondre à des questions de principe. Que ce soit sur des nombres d'heures à effectuer ou sur le montant des factures, il n'est pas possible de trouver un critère qui soit praticable et applicable à court terme dans le cadre de cette ordonnance. C'est pourquoi il est renoncé à prévoir une réglementation en la matière, ce d'autant plus que les dispositions légales ne prévoient pas non plus que l'exercice d'une activité à plein temps soit une condition pour qu'une personne puisse exercer à titre indépendant. En revanche, les cantons sont libres de tenir compte, dans le cadre de leur politique d'autorisation, du fait qu'une activité est exercée à plein temps ou à temps partiel; ils sont même invités à le faire. [mise en exergue par la FMH] [7]

\section{Les conséquences en bref: recommandations pratiques}

1. Le médecin qui est au bénéfice d'une admission et qui peut réaliser son projet de cabinet privé avant le 3 janvier 2006 n'aura à coup sûr aucun problème.

2. Le médecin qui est au bénéfice d'une admission mais qui ne pourra réaliser son projet de cabinet privé qu'à une date ultérieure, doit prendre contact avec la direction de la santé publique de son canton [8] et exiger une prise de position écrite à caractère contraignant. Pour mémoire: les cantons sont libres de choisir les disciplines auxquelles ils vont appliquer le blocage de l'accès à la pratique privée - ils ont donc les pleins pouvoirs en la matière. Ils peuvent aussi étendre au 3 juillet 2006, de manière générale pour tous les médecins, le délai avant lequel un cabinet doit être ouvert et même prolonger ce délai dans des cas individuels.

Par conséquent, ce qui est effectivement applicable n'est pas dicté par le Conseil fédéral mais par les cantons.

3. Le médecin qui est au bénéfice d'une admission mais qui n'a pas encore ouvert son propre cabinet ni ne parvient à obtenir du canton une garantie satisfaisante lui donnant la sécurité nécessaire pour son projet, doit entreprendre les démarches que nous avons mentionnées à plusieurs occasions. Ceci lui permettra d'assurer sa situation aussi bien que possible au niveau juridique pour le cas d'un litige éventuel: il lui faut donc traiter quelques patients en son propre nom et à son propre compte avant l'expiration du délai général (c'est-à-dire d'ici au 3 janvier ou au 3 juillet 2006 selon les cantons).

4. Le médecin qui n'est pas au bénéfice d'une admission peut, selon la volonté du Parlement et aussi selon la pratique observée par son canton, obtenir une admission personnelle s'il s'établit dans des régions dotées d'une couverture sanitaire insuffisante ou s'il reprend l'admission d'un médecin qui lui remet son cabinet.

Nous avons déjà indiqué ci-avant que, conformément à la volonté du Parlement, la reprise d'un cabinet individuel par deux médecins à temps partiel devrait être possible. 